\title{
Avaliação dos impactos da colheita florestal nos atributos físicos do solo em uma reserva legal
}

\author{
Assessment of the impacts of forest harvesting \\ on soil physical attributes of a legal reserve
}

\author{
João Carlos Teixeira Mendes ${ }^{1}$ e Fernando Seixas²
}

\begin{abstract}
Resumo
Este estudo avaliou os efeitos da colheita florestal nos atributos físicos do solo num talhão de Eucalyptus saligna Smith. abandonado há 40 anos. Os três tratamentos testados foram: CCV - colheita convencional com extração mecanizada; CIR1 - colheita de impacto reduzido com extração mecanizada; e CIR2 - coIheita de impacto reduzido com extração animal para lenha (DAP $\leq 30 \mathrm{~cm}$ ) e mecanizada para tora (DAP $>30 \mathrm{~cm}$ ). As variáveis testadas foram: (i) quatro classes de distúrbios superficiais $\left(\mathrm{m}^{2}\right.$.ha-1): sem distúrbio (testemunha), leve, moderado e severo; (ii) resistência do solo à penetração ( $\mathrm{MPa}$ ); e (iii) variação da densidade aparente $\left(\mathrm{g} \mathrm{cm}^{-3}\right)$. Os resultados mostraram que ocorreu compactação significativa do solo quando se fez extração mecanizada de madeira e em áreas onde ocorreram distúrbios moderado e severo. Os tratamentos CIR1 e CIR2 apresentaram médias menores de áreas com distúrbios superficiais moderado e severo, com $1.151 \mathrm{~m}^{2}$.ha-1 e $1.094 \mathrm{~m}^{2}$.ha-1 respectivamente, diferenciando-os estatisticamente do CCV que apresentou $2.620 \mathrm{~m}^{2}$.ha-1. $\mathrm{O}$ uso de sistema de colheita de impacto reduzido minimizou significativamente a área total com compactação de solo prejudicial para o desenvolvimento das plantas e, por isso, é alternativa viável do ponto de vista ambiental para a extração de madeira em talhões abandonados de Eucalyptus e para o manejo florestal em reserva legal.
\end{abstract}

Palavras-chave: Colheita de impacto reduzido; Extração animal; Reserva legal; Colheita florestal.

\begin{abstract}
The objective of this study was to assess the effects of forest harvesting on physical attributes of the soil in a stand of Eucalyptus saligna Smith. abandoned for 40 years. The three treatments were: CCV conventional harvesting with mechanized extraction; CIR1 - reduced impact harvesting with mechanized extraction; and CIR2 - reduced impact harvesting for firewood associated with animal extraction (DBH $\leq$ $30 \mathrm{~cm}$ ) and with mechanized extraction for logs (DAP > $30 \mathrm{~cm}$ ). The variables of soil effects were: (i) four classes of surface disturbances $\left(\mathrm{m}^{2} . \mathrm{ha}^{-1}\right)$ : "no-disturbances", "light", "moderate" and "severe disturbances"; (ii) cone penetrometer resistance (MPa); and (iii) bulk density variation $\left(\mathrm{g} \cdot \mathrm{cm}^{-3}\right)$. Soil compaction was detected at mechanized extraction and in areas with moderate and severe disturbances. The treatments CIR1 and CIR2 had lower averages of moderate and severe disturbances; $1.151 \mathrm{~m}^{2}$.ha-1 and $1.094 \mathrm{~m}^{2}$.ha-1 respectively, differentiating them statistically from CCV that presented $2.620 \mathrm{~m}^{2}$. ha- ${ }^{-1}$. The use of a reduced impact harvesting system significantly minimized the total area with critical soil compaction for plants development and, therefore, is an environmentally viable alternative to logging in abandoned stands of Eucalyptus and for forest management in legal reserve.
\end{abstract}

Keywords: Reduced impact harvesting; Animal skidding; Legal reserve; Forest harvesting

\section{INTRODUÇÃO}

O abandono ou o pousio por longo tempo de monocultivos com determinadas espécies arbóreas proporciona condições favoráveis para a regeneração de outras espécies no sub-bosque, em função da cessação do manejo silvicultural e das intervenções antrópicas no interior do povoamento. Embora as constatações científicas tenham mostrado que o abandono das antigas unidades produtivas favorecem a dinâmica da vegetação natural no sub-bosque e o habitat para espécies da fauna silvestre, alguns autores recomendam a subtração de árvores de eucalipto visando diminuir

${ }^{1}$ Doutor em Recursos Florestais. USP - Universidade de São Paulo. ESALQ - Escola Superior de Agricultura "Luiz de Queiroz". Horto Florestal de Itatinga. Caixa Postal 34 - 18690-000 - Itatinga, SP, Brasil. E-mail: jctmende@usp.br.

2Professor Doutor do Departamento de Ciências Florestais. USP - Universidade de São Paulo. ESALQ - Escola Superior de Agricultura "Luiz de Queiroz". Av. Pádua Dias, 11 - Caixa postal 09 - 13418-900 - Piracicaba, SP - Brasil. E-mail: fseixas@usp.br. 
a competição da espécie exótica com as espécies nativas e, assim, contribuir para o processo de restabelecimento da vegetação natural (CARNEIRO, 2002; GONÇALVES et al., 2010; ONOFRE, 2009; RODRIGUES et al., 2009; SAPORETTI JÚNIOR et al., 2003; GONÇALVES et al., 2010).

Segundo as disposições legais no inciso III do Artigo $3^{\circ}$. da Lei 12.651/2012 (BRASIL, 2012) de 25 de maio de 2012, a reserva legal na propriedade, além de suas funções ambientais, tem como objetivo assegurar o uso econômico de modo sustentável dos recursos naturais do imóvel rural. Como parte das diretrizes e recomendações para o manejo florestal sustentável, previstas no inciso III do Artigo 22, recomenda-se o manejo de espécies exóticas com adoção de medidas que favoreçam a regeneração de espécies nativas.

Considerando o amparo legal para utilizar a reserva legal de forma sustentável e, ainda, as recomendações científicas de reduzir a densidade do povoamento da espécie exótica em prol da regeneração do sub-bosque das florestas abandonadas de Eucalyptus, infere-se que há forte tendência para que essas antigas unidades de produção florestal sejam revertidas em reserva legal, visando principalmente o cumprimento da exigência legal em concomitância com o aproveitamento econômico do uso da terra.

Como parte do processo de conversão de antigas unidades de produção de madeira em reserva legal, o grande desafio é aproveitar o seu potencial econômico com o mínimo de prejuízo ambiental. Um dos entraves para o aproveitamento econômico do Eucalyptus são as alterações ambientais inevitáveis da colheita florestal (MALINOVSKI et al, 2008).

Estudos têm demonstrado que a frequência e a intensidade de tráfego de máquinas da colheita florestal no interior do povoamento são fatores que alteram os atributos físicos do solo, podendo atingir níveis críticos de compactação para o desenvolvimento radicular das plantas remanescentes (LOPES et al., 2015; SEIXAS et al., 1998; SZYMCZAK et al., 2014).

O tráfego de máquinas também promove distúrbios significativos na superfície do solo (SEIXAS et al., 2003). As alterações superficiais podem promover a remoção total da cobertura vegetal e, consequentemente, aumentar a área de solo exposto aos fatores de erosão e de compactação. A manutenção da cobertura vegetal na superfície é importante porque o tráfego de máquinas sobre camadas de resíduos vegetais diminui o nível de compactação do solo (LOPES et al., 2015; SEIXAS et al., 1998; SZYMCZAK et al., 2014). O uso de tração animal na extração de madeira ao invés de tração mecânica é outra medida que pode minimizar os danos na superfície do solo (DEAL, 2002; FICKLIIN et al., 1997).

Rodrigues et al. (2009) recomendaram a retirada da espécie exótica de florestas abandonadas adotando-se técnicas de colheita florestal de impacto reduzido. O direcionamento do corte e da queda das árvores sob a supervisão e a orientação técnica da equipe operacional são medidas recomendadas para mitigar os impactos da colheita florestal, principalmente à floresta remanescente $e$ ao solo (AKAY et al., 2006; CLATTERBUCK, 2006; JOHNS et al.,1998; MEADOWS, 1993; PINTO et al., 2002; SABOGAL et al., 2000).

Holmes et al. (2004) constataram o dobro de área impactada com a colheita convencional quando comparada com a colheita de impacto reduzido. Nesse mesmo estudo, 100\% das trilhas de tratores utilizadas na colheita convencional apresentaram exposição de solo mineral enquanto que nas trilhas da colheita de impacto reduzido apenas $10 \%$ apresentaram o mesmo tipo de impacto.

De acordo com FAO (2004), o sistema de colheita de impacto reduzido tem se mostrado como uma alternativa viável para o manejo florestal sustentável. Embora esse sistema de colheita já tenha sido comprovado como o melhor método para o caso das florestas tropicais (BARROS; UHL, 1995; HOLMES et al., 2002; JOHNS et al., 1998; MINETTI et al., 2000; VALLE et al., 2007), a sua viabilidade como alternativa para a extração de árvores de Eucalyptus em áreas abandonadas com presença de sub-bosque tem sido pouco testada.

Nesse sentido, este trabalho avaliou os efeitos nos atributos físicos do solo de diferentes sistemas de colheita da madeira num plantio abandonado de Eucalyptus numa reserva legal.

\section{MATERIAL E MÉTODOS}

Este experimento foi desenvolvido num talhão plantado com Eucalyptus saligna Smith com área total de 33,0 ha, que se encontra sem manejo há mais de 40 anos e faz parte das áreas destinadas 
à reserva legal da Estação Experimental de Ciências Florestais da Universidade de São Paulo (USP), no município de Itatinga/SP $\left(23^{\circ} 00^{\prime} 55^{\prime \prime}\right.$ S e $\left.48^{\circ} 39^{\prime} 57 \mathrm{~W}\right)$.

O relevo é predominantemente plano, com variação entre $2 \%$ e $10 \%$ de declividade principalmente nas extremidades do talhão. O solo foi classificado como Latossolo Vermelho-Amarelo Distrófico típico A moderado com textura média (LVAd) (GONÇALVES et al., 2012). Os resultados das análises físicas das amostras coletadas até $30 \mathrm{~cm}$ de profundidade apresentaram concentração média de $85 \%$ de areia total e $10 \%$ de argila e valores médios da densidade aparente entre 1,53 a 1,56 g. $\mathrm{cm}^{-3}$, obtidos com $18 \%$ de teor médio de água no solo.

No sub-bosque do povoamento de Eucalyptus saligna há regeneração natural de árvores e arbustos de espécies nativas representativas da flora de cerrado e de floresta estacional semidecidual, indicando uma regeneração de vegetação de transição ou cerradão. No início do estudo foi realizado o inventário $100 \%$ em sistemas de faixas em $25 \%$ da área do talhão ou 8,37 ha, onde mediu-se a altura e o diâmetro de 3.392 indivíduos de Eucalyptus e de 4.452 indivíduos de espécies nativas com diâmetro a altura do peito (DAP) a partir de $5,0 \mathrm{~cm}$.

Os indivíduos plaquetados foram georreferenciados com a transformação das suas coordenadas cartesianas (x e y) em coordenadas UTM. Com as ferramentas do software ArcGis 9.2 efetuou-se a interpolação dos valores do inventário de cada indivíduo e foram gerados mapas do talhão com três classes de volume $\left(\mathrm{m}^{3}\right)$ e de área basal $\left(\mathrm{m}^{2} . \mathrm{ha}^{-1}\right)$. Com isso constatou-se visualmente a heterogeneidade espacial de volume e de área basal existente ao longo do talhão, tanto na população de Eucalyptus quanto na população de espécies nativas do sub-bosque. A aplicação do teste de correlação de Pearson mostrou correlação inversa entre os parâmetros das duas populações, ou seja, no sítio com maior volume e área basal de Eucalyptus ocorre menor densidade e área basal de árvores nativas e vice-versa.

\section{Delineamento Experimental}

Com a constatação da heterogeneidade espacial de volume e de área basal nas populações e da correlação inversa entre elas, a partir da análise dos mapas das classes de volume e de área basal de Eucalyptus, adotou-se o delineamento experimental de blocos ao acaso (PIMENTEL-GOMES; GARCIA, 2002), cujos blocos representaram áreas com alto, médio e baixo valor de área basal e de volume de Eucalyptus.

Os três tratamentos testados foram: CCV - colheita convencional com extração mecanizada; CIR1 - colheita de impacto reduzido com extração mecanizada; e CIR2 - colheita de impacto reduzido com extração animal para lenha e mecanizada para tora. A área experimental total foi de 2,43 ha, sendo que os três tratamentos foram instalados em faixas e em cada tratamento foram instaladas três parcelas de 30,0 x 30,0 m por bloco, totalizando nove parcelas e área amostral de 0,81 ha por tratamento, conforme observado na Figura 1.

As principais medidas mitigadoras de impacto adotadas nos tratamentos com sistemas de impacto reduzido foram: (i) a abertura planejada do ramal principal de extração de madeira antes do início das operações da colheita florestal, passando por áreas menos densas de vegetação nativa do sub-bosque; (ii) o direcionamento do corte e da queda das árvores de eucalipto; e (iii) a supervisão e a orientação técnica da equipe de colheita. No caso da colheita convencional não houve supervisão técnica da equipe de corte e os ramais de extração foram abertos no decorrer da operação de remoção da madeira.

Nesse estudo extraiu-se $100 \%$ do volume de Eucalyptus em duas etapas. Na etapa 1 foram extraídas as árvores com DAP $<30 \mathrm{~cm}$ para uso na forma de lenha. Já, na etapa 2 foram extraídas as árvores remanescentes com $\mathrm{DAP} \geq 30 \mathrm{~cm}$ na forma de toras para serraria. A colheita em duas etapas foi essencial tanto para o controle experimental quanto para o melhor aproveitamento da lenha e da tora. Antes da colheita, o volume médio de madeira de Eucalyptus e os parâmetros médios estruturais da vegetação nativa do sub-bosque não demonstraram diferenças estatísticas entre as áreas experimentais dos três tratamentos, conforme observado na Tabela 1. 


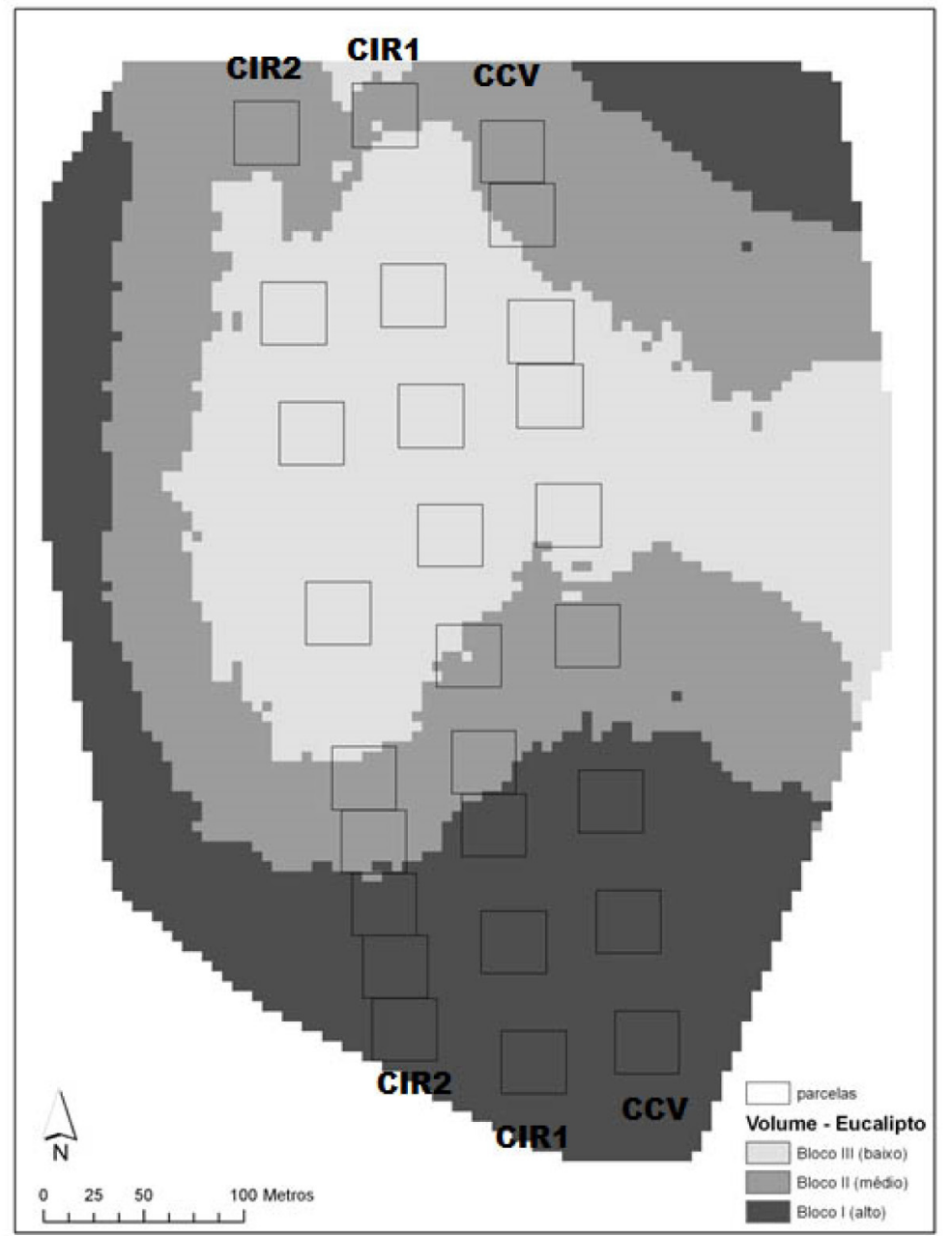

Figura 1. Distribuição espacial dos blocos e das parcelas em cada tratamento.

Figure 1. Spatial distribution of blocks and plots in each treatment.

Tabela 1. Valores médios de parâmetros estruturais do povoamento de Eucalyptus saligna e da vegetação nativa do sub-bosque, antes da colheita florestal.

Table 1. Average values of structural parameters of the understory at the Eucalyptus saligna and native vegetation stands, before forest harvesting.

\begin{tabular}{lccc}
\hline \multirow{2}{*}{ Tratamentos } & Eucalyptus saligna & \multicolumn{2}{c}{ Espécies Nativas } \\
\cline { 2 - 4 } & Volume & Vivas & Área basal \\
\hline CCV & $\mathbf{m}^{3} \cdot \mathbf{h a}^{-1}$ & indivíduos.ha-1 $^{-1}$ & $\mathbf{m}^{\mathbf{2}} \cdot \mathbf{h a}^{-1}$ \\
CIR1 & $289,61(\mathrm{a})$ & $547(\mathrm{a})$ & $6,77(\mathrm{a})$ \\
CIR2 & $277,57(\mathrm{a})$ & $526(\mathrm{a})$ & $7,21(\mathrm{a})$ \\
\hline
\end{tabular}

Nota: CCV - Colheita Convencional; CIRI - Colheita de Impacto Reduzido I; CIR2 - Colheita de Impacto Reduzido 2; ind. - indivíduos. Médias seguidas de letras iguais na mesma coluna não diferem estatisticamente pelo teste de Tukey $(p<0,05)$

As atividades e os equipamentos utilizados em cada tratamento e em cada etapa de colheita estão descritos na Tabela 2.

Nos tratamentos CCV e CIR1, as árvores foram cortadas com motosserra. Nesses dois tratamentos a lenha foi extraída até o pátio utilizando-se um conjunto trator-carreta agrícola, com uma carga média de $10,0 \mathrm{~m}^{3}$ por viagem. No tratamento CIR2, inicialmente as árvores foram cortadas com uso de motosserra, sendo em seguida a lenha extraída com o conjunto carroça-tração animal até a margem do carreador, com uma carga média de $1,2 \mathrm{~m}^{3}$ por viagem. A lenha foi extraída com comprimento entre 1,0 m e 1,3 m, tanto na extração mecânica quanto na extração com tração animal. Já, no tratamento com colheita convencional (CCV) e nos dois tratamentos de colheita de impacto reduzido (CIR1 e CIR2), as toras foram cortadas com motosserra e extraídas no comprimento médio de $15 \mathrm{~m}$, por meio de arraste para fora do talhão até o pátio utilizando-se trator agrícola (128 $\mathrm{kW}$ ) equipado com pinça hidráulica traseira (Mini-Skidder) com garra de abertura máxima de 1,6 $\mathrm{m}$, (Figura 2). 
Tabela 2. Recursos operacionais utilizados para a colheita florestal em cada sistema de colheita de cada tratamento. Table 2. Operational resources used for forest harvesting in each system and treatment.

\begin{tabular}{|c|c|c|c|c|c|c|}
\hline \multirow{2}{*}{$\begin{array}{l}\text { Etapas } \\
\text { de colheita }\end{array}$} & \multirow{2}{*}{ Atividades } & \multirow{2}{*}{$\begin{array}{c}\text { Máquinas e } \\
\text { Equipamento }\end{array}$} & \multirow{2}{*}{ Especificações } & \multicolumn{3}{|c|}{ Tratamentos } \\
\hline & & & & $\mathrm{CCV}$ & CIR1 & CIR2 \\
\hline \multirow{3}{*}{$\begin{array}{l}\text { Etapa } 1 \\
\text { Colheita } \\
\text { de lenha }\end{array}$} & 1. Corte & Motosserra & $\begin{array}{l}\text { Marca STHIL (MS381) } \\
3,9 \mathrm{~kW}\end{array}$ & $x$ & $x$ & $x$ \\
\hline & \multirow{2}{*}{ 2. Extração } & $\begin{array}{c}\text { Conjunto } \\
\text { Trator - carreta agrícola }\end{array}$ & $\begin{array}{l}\text { Trator: Marca Ford, ano 1962, 4x2 e } 60 \text { kW; } \\
\text { Carreta agrícola com carga média de } 14,0 \mathrm{~m}^{3}\end{array}$ & $x$ & $x$ & \\
\hline & & $\begin{array}{c}\text { Conjunto } \\
\text { Tração animal - carroça }\end{array}$ & $\begin{array}{l}\text { Animal: cavalo sem raça definida } \\
\text { Carroça com carga média de } 1,2 \mathrm{~m}^{3}\end{array}$ & & & $x$ \\
\hline \multirow[b]{2}{*}{$\begin{array}{l}\text { Etapa } 2 \\
\text { Colheita } \\
\text { de tora }\end{array}$} & 3. Corte & Motosserra & $\begin{array}{l}\text { Marca STHIL (MS660) } \\
5,2 \mathrm{~kW}\end{array}$ & $x$ & $x$ & $x$ \\
\hline & 4. Extração & $\begin{array}{c}\text { Conjunto } \\
\text { Trator - } \\
\text { Pinça hidráulica } \\
\text { (Mini-Skidder) }\end{array}$ & $\begin{array}{l}\text { Trator: Marca Valmet, ano } 1990,4 \times 4 \text { e } \\
128 \text { kW } \\
\text { Mini-Skidder com garra de } 1,6 \text { m de aber- } \\
\text { tura máxima }\end{array}$ & $x$ & $x$ & $x$ \\
\hline
\end{tabular}

Nota: CCV - Colheita Convencional; CIRI - Colheita de Impacto Reduzido I; CIR2 - Colheita de Impacto Reduzido 2
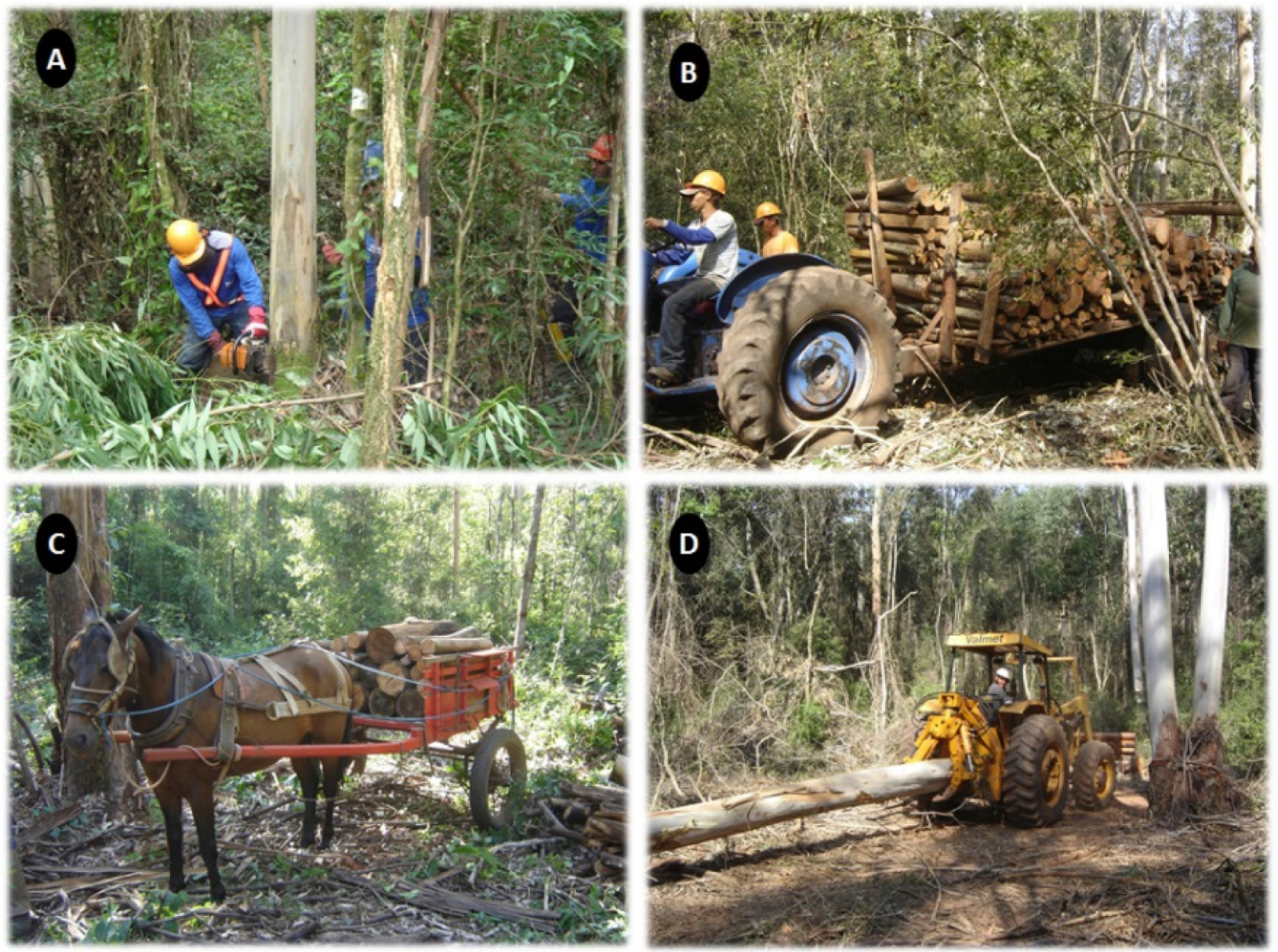

Figura 2. Modos de colheita florestal: (A) corte com motosserra; (B) extração mecanizada na etapa 1 (colheita de lenha); (C) extração com animal na etapa 1 (colheita de lenha); (D) extração mecanizada na etapa 2 (colheita de tora).

Figure 2. Forest logging systems: (A) cut with chainsaws; (B) mechanized extraction in step 1 (wood harvesting); (C) with an animal extraction in step 1 (wood harvesting); (D) mechanized extraction in step 2 (log harvesting).

As avaliações dos impactos provenientes da colheita florestal nos atributos físicos do solo foram realizadas ao término de cada etapa de colheita, ou seja, após a extração do volume total de lenha e após a extração do volume total de tora.

\section{Variáveis dos atributos físicos do solo}

No estudo dos impactos nos atributos físicos do solo avaliaram-se os distúrbios superficiais, a resistência do solo à penetração e a variação da densidade aparente. O método de avaliação dos 
distúrbios superficiais no solo foi adaptado do método utilizado no estudo de Seixas et al. (2003), adotando-se quatro classes de distúrbios conforme descrito a seguir:

Classe 1 - sem distúrbio (testemunha).

Classe 2 - distúrbio leve: sem remoção da cobertura vegetal no solo e sem promover a remoção de serapilheira.

Classe 3 - distúrbio moderado: com remoção da cobertura vegetal do solo e com remoção de serapilheira. Classe 4 - distúrbio severo: com remoção da cobertura vegetal do solo e com exposição do solo mineral (Figura 3).

Em cada parcela foi instalada uma malha de barbante com quadrículas de 2,0 x 1,0 m, totalizando 450 quadrículas de $2,0 \mathrm{~m}^{2}$ por parcela, ou seja, 4.050 quadrículas por tratamento. Considerando a área experimental dos três tratamentos foram avaliados 12.150 quadrículas em cada etapa de colheita. No centro de cada quadrícula foi atribuída uma classe de distúrbio superficial predominante por meio de análise visual e a sua localização foi georreferenciada em coordenadas UTM. Com as ferramentas do software ArcGis 9.2 efetuou-se o cálculo da área $\left(\mathrm{m}^{2} . \mathrm{ha}^{-1}\right)$ de cada classe de distúrbio por tratamento e, também, geraram-se mapas para a interpretação da distribuição espacial dos distúrbios.

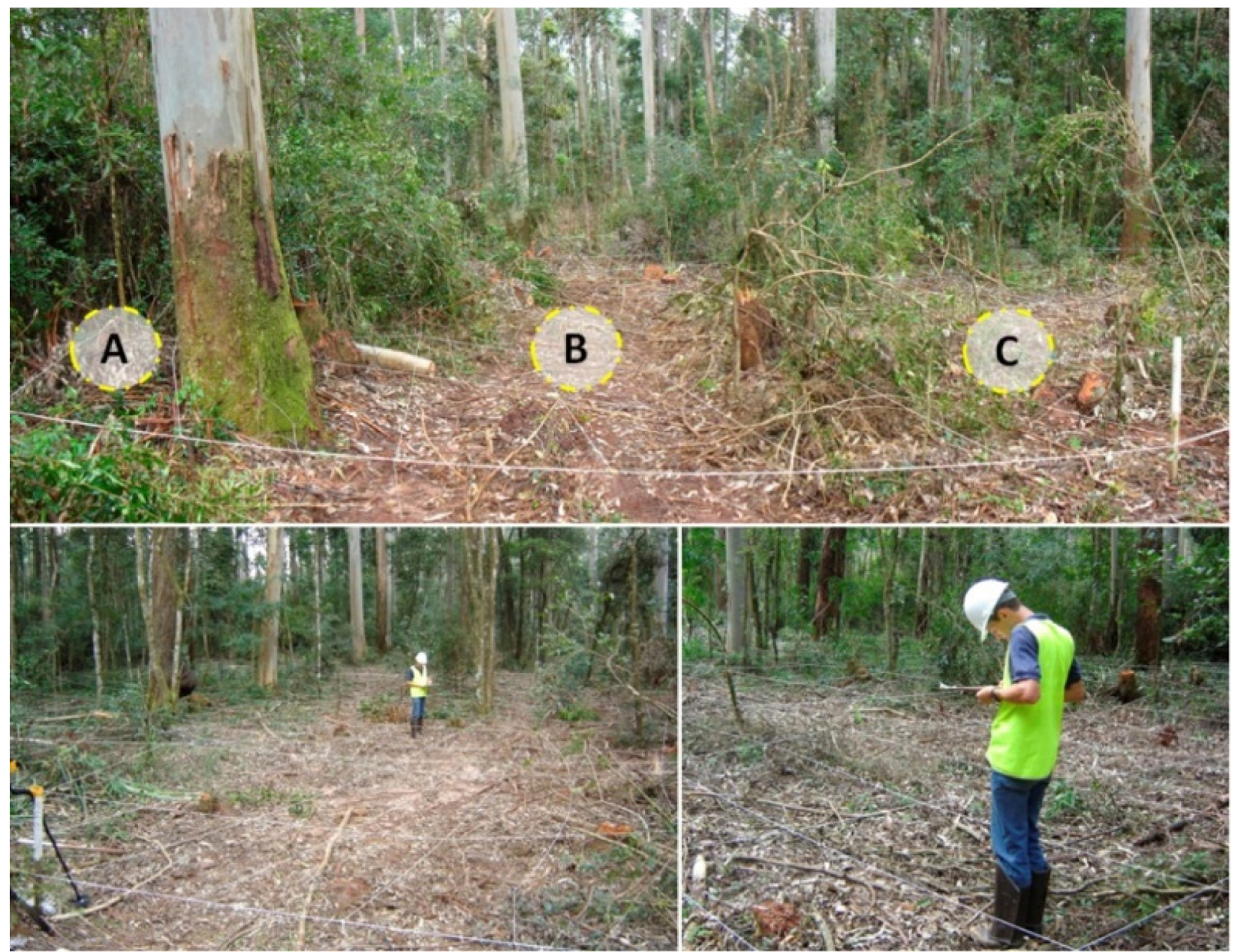

Figura 3. Interior do talhão após a colheita de lenha no CIR1: (A) região sem distúrbio; (B) região com distúrbios moderado e severo; (C) região com distúrbio leve; avalição dos danos na superfície.

Figure 3. Inside the stand after wood harvest at CIR1: (A) region no-disturbances; (B) region with moderate and severe disturbances; (C) region with light disturbance; Assessment of surface disturbances.

A densidade aparente do solo (Ds), antes e após a colheita, foi medida a partir de amostras de solo obtidas com martelo deslizante AMS e anel metálico de $5 \mathrm{~cm}$ de comprimento, em cada uma das seguintes faixas de profundidade: 5 a $10 \mathrm{~cm} ; 15$ a $20 \mathrm{~cm} ; 25$ a $30 \mathrm{~cm}$; e 35 a $40 \mathrm{~cm}$. Essa amostragem foi realizada em uma quadrícula de cada classe de distúrbios superficiais por parcela, totalizando em cada tratamento e em cada etapa 36 amostras por profundidade. As amostras de solo foram secas em estufa a $105^{\circ} \mathrm{C}$ por 24 horas e foram pesadas antes e após a secagem para determinar o teor de água no solo. Nesse estudo adotou-se um aumento de $15 \%$ a $20 \%$ no valor inicial da densi- 
dade do solo como nível crítico de compactação do solo (Geist et. al., 1989 apud SEIXAS; OLIVEIRA JUNIOR, 2001). A equação utilizada para determinar a densidade aparente foi:

$$
\text { Ds }\left(\text { g. } \mathrm{cm}^{-3}\right)=\operatorname{Mss}(\mathrm{g}) / \mathrm{Vt}\left(\mathrm{cm}^{-3}\right)
$$

Ds = densidade aparente do solo

Mss = massa do solo seco

$\mathrm{Vt}=$ volume total do anel metálico

A resistência do solo à penetração $(\mathrm{R})$ foi determinada por meio de penetrômetro de impacto, modelo IAA/Planalsucar (STOLF, 1991), até $40 \mathrm{~cm}$ de profundidade. As medições foram realizadas em 5 quadrículas de cada classe de distúrbio superficial por parcela, totalizando 20 pontos amostrais que foram sorteados entre as 450 quadrículas de cada parcela. Ou seja, foram avaliados 180 pontos amostrais por tratamento em cada etapa. O número de impactos do penetrômetro através do perfil do solo foi transformado em MPa, por meio da multiplicação por 0,1 do valor resultante da equação desenvolvida por Stolf (1991):

$$
R\left(\mathrm{kgf.} \mathrm{cm}^{-2}\right)=5,6+6,89 * N(\text { impactos } / \mathrm{dm})
$$

O limite crítico de resistência à penetração (RP) ou compactação para o desenvolvimento radicular das plantas adotado nesse estudo foi de 2,5 a 3,0 MPa (Sands et al., 1979 apud SEIXAS; SOUZA, 2007).

\section{Análise Estatística}

Todos os valores calculados foram submetidos à Análise de Variância (ANOVA) e as médias comparadas pelo teste de Tukey. Os parâmetros dos modelos, tais como homogeneidade de variâncias e distribuição normal dos resíduos, foram checados pelo teste de Bartlett e Shapiro-Wilk, sendo realizada a transformação dos dados quando necessário. Também foi realizado o teste de correlação de Pearson ( $\mathrm{r}$ ) para algumas variáveis. Todas as análises foram realizadas usando o software R versão 2.15.1.

\section{RESULTADOS E DISCUSSÃO}

O volume total de madeira de Eucalyptus saligna extraído foi de $1312,30 \mathrm{~m}^{3}$, sendo $343,97 \mathrm{~m}^{3}$ na forma de lenha e $968,33 \mathrm{~m}^{3}$ na forma de tora, $26 \%$ e $74 \%$ do volume total respectivamente. O volume médio de madeira extraída em cada tratamento foi de $289,61 \mathrm{~m}^{3} \cdot \mathrm{ha}^{-1}$ no CCV, $277,57 \mathrm{~m}^{3} \cdot \mathrm{ha}^{-1}$ no CIR1 e 316,37 $\mathrm{m}^{3}$.ha ${ }^{-1}$ no CIR2. O teste de Tukey $(\mathrm{p}<0,05)$ comprovou não existir diferença estatística entre os volumes médios de lenha e de tora que foram extraídos nos tratamentos.

\section{Efeitos da colheita nos atributos físicos do solo}

Os resultados apresentados na Tabela 3 demonstram que a colheita florestal promoveu distúrbios significativos na superfície do solo tanto pelo método convencional quanto de impacto reduzido. Os distúrbios superficiais no solo se agravaram na etapa 2, principalmente, devido ao maior volume de madeira extraída na forma de toras e que exigiu maior intensidade de tráfego de máquinas no interior do povoamento. Somando-se os valores das classes de distúrbios moderado e severo, os aumentos relativos de área por hectare entre a etapa 1 e a etapa 2 foram de $138 \%$ no CCV, de $125 \%$ no CIR1 e de $236 \%$ no CIR2.

Muitas áreas remanescentes sem distúrbio da etapa 1 sofreram alterações superficiais na etapa 2, principalmente devido ao maior número de manobras necessárias para agarrar e arrastar as toras para fora do talhão. No caso do CCV e do CIR1, a extração de madeira com tração mecânica na etapa 1 promoveu a abertura de ramais secundários e, oportunamente, na etapa 2 esses ramais serviram de orientação ao operador de máquina e facilitaram as manobras para a extração das toras.

Já, no caso do CIR2, o uso de tração animal para a extração de lenha praticamente não promoveu abertura de ramais secundários. Isso se tornou um fator complicador para as manobras e o deslocamento da máquina na extração de toras devido à densidade de plantas do sub-bosque e, por isso, foi necessário maior movimentação do trator no interior do talhão. Consequentemente, em relação aos resultados do CCV e do CIR1, houve aumento relativo bem maior de áreas com distúrbios moderado e severo entre a etapa 1 e a etapa 2 do CIR2. 
Mendes et al. - Avaliação dos impactos da colheita florestal nos atributos físicos do solo em uma reserva legal

Tabela 3. Área média de distúrbio na superfície do solo por tratamento, medida após as colheitas de madeira nas etapas 1 e 2.

Table 3. Average area disturbance on the soil surface by treatment measured after harvesting wood in steps 1 and 2.

\begin{tabular}{lccc|ccc}
\hline & \multicolumn{5}{c}{ Tratamentos } \\
\cline { 2 - 7 } Classes de distúrbios superficiais & $\mathbf{C C V}$ & CIR1 & CIR2 & CCV & CIR1 & CIR2 \\
\cline { 2 - 7 } & \multicolumn{7}{c}{ m $^{2}$.ha-1 } \\
\cline { 2 - 7 } & \multicolumn{2}{c}{ Etapa 1 (colheita de lenha) } & \multicolumn{2}{c}{ Etapa 2 (colheita de tora) } \\
\hline Sem Distúrbio & $7140 \mathrm{Aa}$ & $7316 \mathrm{Aa}$ & $7476 \mathrm{Aa}$ & $3469 \mathrm{Ab}$ & $5363 \mathrm{Aa}$ & $4153 \mathrm{Aab}$ \\
Leve & $1758 \mathrm{Ba}$ & $2172 \mathrm{Ba}$ & $2197 \mathrm{Ba}$ & $3911 \mathrm{Aa}$ & $3486 \mathrm{Aa}$ & $4753 \mathrm{Aa}$ \\
Moderado & $748 \mathrm{Ca}$ & $405 \mathrm{Cb}$ & $291 \mathrm{Cb}$ & $1649 \mathrm{Ba}$ & $896 \mathrm{Bb}$ & $688 \mathrm{Bb}$ \\
Severo & $353 \mathrm{Da}$ & $106 \mathrm{Db}$ & $34 \mathrm{Db}$ & $971 \mathrm{Ba}$ & $255 \mathrm{Bb}$ & $406 \mathrm{Bb}$ \\
\hline
\end{tabular}

Nota: CCV - Colheita Convencional; CIRI - Colheita de Impacto Reduzido I; CIR2 - Colheita de Impacto Reduzido 2. Letras iguais, minúsculas na mesma linha e maiúsculas na mesma coluna, indicam que os valores médios dos tratamentos não diferem estatisticamente pelo teste de Tukey $(p<0,05)$. Comparações somente na mesma etapa.

Comparando os resultados dos dois tratamentos com colheita de impacto reduzido apresentados na Tabela 3, nota-se que não houve diferença significativa entre as médias de áreas com distúrbios moderado e severo na superfície do solo, em nenhuma das etapas. Porém, é certo afirmar que na etapa 1 a extração de lenha com tração animal minimizou significativamente os efeitos negativos na superfície do solo, uma vez que em relação ao CIR1, os cálculos demonstram que o CIR2 apresentou redução relativa de área média por hectare com distúrbios moderado e severo de $39 \%$ e $212 \%$, respectivamente. A minimização dos efeitos na superfície do solo durante a extração da madeira com tração animal também foi constatada por Deal (2002) e Fickliin et al. (1997)

Nota-se na Figura 4 que além de ter ocorrido minimização dos distúrbios na superfície no CIR2, a limitação de áreas favoráveis ao tráfego do animal no interior do povoamento promoveu a concentração dos distúrbios nos arredores do ramal principal de extração de madeira. Apesar do uso de tração mecânica, no CIR1 também houve concentração da distribuição espacial dos distúrbios superficiais no ramal principal, porém, de forma menos acentuada que no CIR2. A concentração da distribuição espacial dos distúrbios superficiais tem como vantagem o menor impacto visual e a melhoria do aspecto social considerando que as alterações estéticas na natureza são um dos efeitos de maior destaque da colheita florestal (SEIXAS, 2002). Portanto, conclui-se que os tratamentos com colheita de impacto reduzido promoveram menor impacto visual do que o tratamento com colheita convencional.

Reportando-se aos resultados da Tabela 3, nota-se que com os métodos de colheita de impacto reduzido foi possível minimizar a geração de distúrbios superficiais das classes moderado e severo. Nas duas etapas, os valores médios por hectare desses distúrbios dos tratamentos com colheita de impacto reduzido diferenciaram-se estatisticamente do tratamento com colheita convencional.

No final da etapa 2, os valores médios somando-se as classes de distúrbios moderado e severo nos CIR1 e CIR2 foram de $1.151 \mathrm{~m}^{2}$.ha-1 e $1.094 \mathrm{~m}^{2} \cdot \mathrm{ha}^{-1}$, respectivamente, enquanto que no CCV foi de $2.620 \mathrm{~m}^{2}$.ha-1. Comparando-se com o CCV, após a colheita total de madeira de Eucalyptus, os sistemas de colheita CIR1 e CIR2 reduziram relativamente os valores de área média por hectare com distúrbios moderado e severo em 171\% e 178\%, respectivamente. Essa redução foi muito importante porque nessas áreas também foram constatados níveis críticos de compactação do solo.

Os resultados das análises de densidade aparente de solo mostraram que os dois tratamentos com extração mecanizada de madeira foram os que apresentaram aumentos relativos até o limite crítico. Na etapa 1, o CCV apresentou aumento de 15\% e o CIR1 de 16\% na camada entre 15 a 20 $\mathrm{cm}$ de profundidade em áreas com distúrbio superficial severo. Na etapa 2, constatou-se no CIR1 aumento de $17 \%$ da densidade aparente na profundidade de 15 a $20 \mathrm{~cm}$ em áreas com distúrbio superficial moderado. No caso do CIR2, o aumento relativo de densidade proveniente do tráfego animal atingiu um valor máximo de $6 \%$. Já na etapa 2 do CIR2, apesar de não ter atingido o nível crítico, em áreas com distúrbios superficiais moderado e severo constatou-se aumento de 10\% da densidade até $30 \mathrm{~cm}$ de profundidade. Esses resultados mostram que o tráfego de máquina na colheita florestal é uma das principais fontes de energia que podem causar compactação de solo, corroborando com a afirmativa de Gonçalves (2002). 


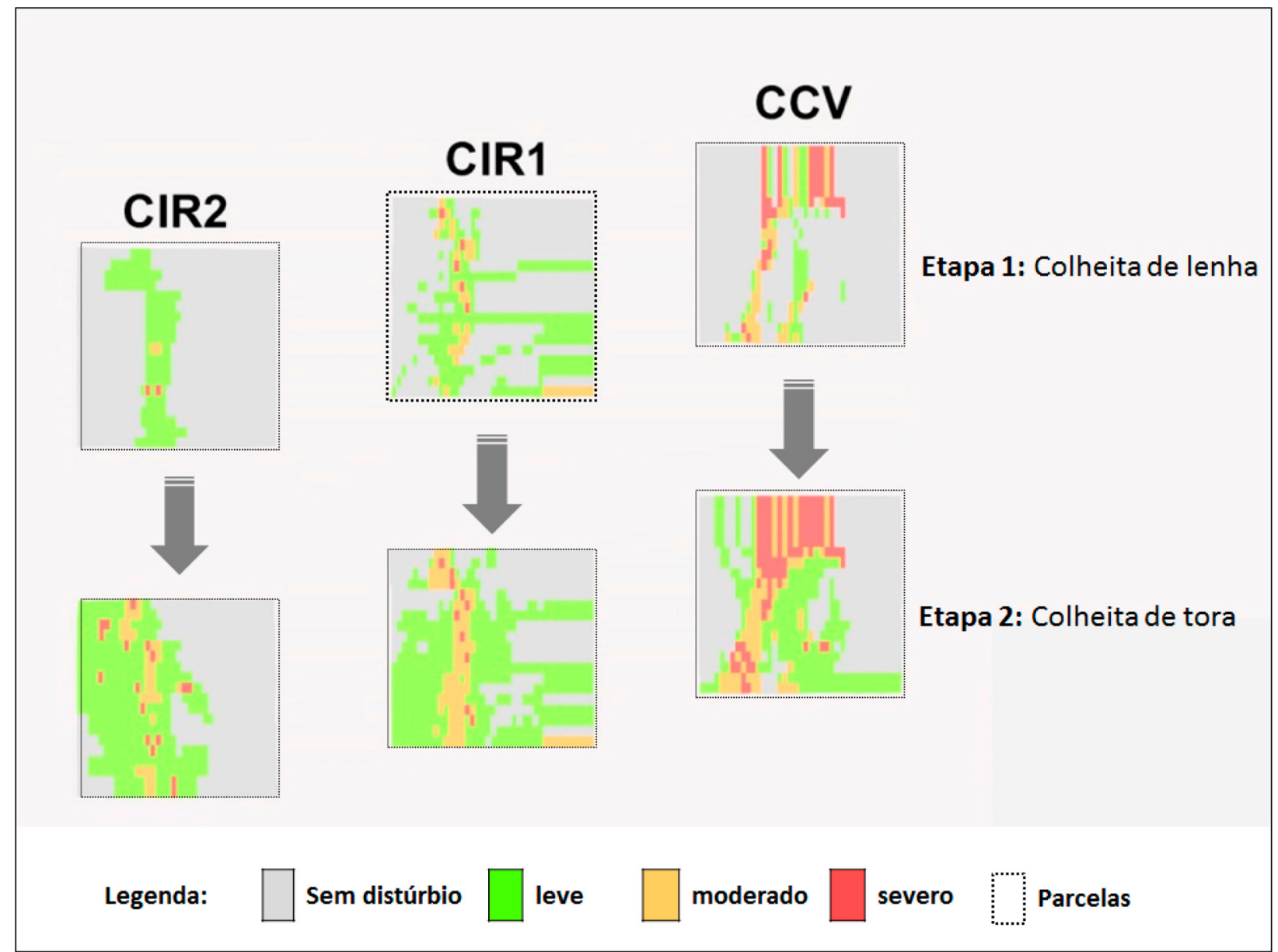

Figura 4. Exemplo da evolução da distribuição espacial dos distúrbios superficiais entre as etapas 1 e 2, nas mesmas parcelas em cada um dos tratamentos. Sendo: CCV - Colheita Convencional; CIR1 - Colheita de Impacto Reduzido 1; CIR2 - Colheita de Impacto Reduzido 2.

Figure 4. Example evolution of the spatial distribution of disturbances surface between steps 1 and 2 , the same plot in each treatment. Where: CCV - conventional harvesting; CIR1 - reduced impact harvesting 1; CIR2 reduced impact harvesting 2.

Os resultados das análises da resistência do solo à penetração (RP) e dos gráficos de índice de cone evidenciam a compactação no solo que ocorreu em cada etapa de colheita florestal.

Na Etapa 1, somente no tratamento CIR2 não foram constatados níveis críticos de resistência à penetração (Figura 5b). O valor médio máximo de RP nesse caso foi de 2,16 Mpa na classe com distúrbio severo, o que indica uma compactação aceitável devido ao valor crítico ser de 2,5 MPa. Comparativamente, no tratamento com colheita convencional os valores médios de RP atingiram a faixa crítica de compactação na camada de solo entre $15 \mathrm{~cm}$ e $35 \mathrm{~cm}$ de profundidade nas áreas com distúrbios moderado e severo (Figura 5a). O aumento da resistência à penetração em profundidade pode estar associado ao teor um pouco maior de argila nas camadas mais profundas do solo da área de estudo, conforme evidenciado por Gonçalves et al. (2012), visto que quanto maior o teor de argila no solo maior será a coesão entre as partículas (GONÇALVES, 2002).

Considerando que não houve diferença entre o volume médio de lenha extraído entre os tratamentos e que as características dos danos na superfície foram semelhantes, conclui-se que o fator diferencial de compactação na Etapa 1 foi o tipo de tração utilizada na extração de lenha. Enquanto que a pressão exercida pelo módulo com tração animal promoveu compactação abaixo do limite crítico de resistência à penetração, a pressão exercida pelo módulo com tração mecânica foi suficiente para compactar o solo além do limite crítico. 


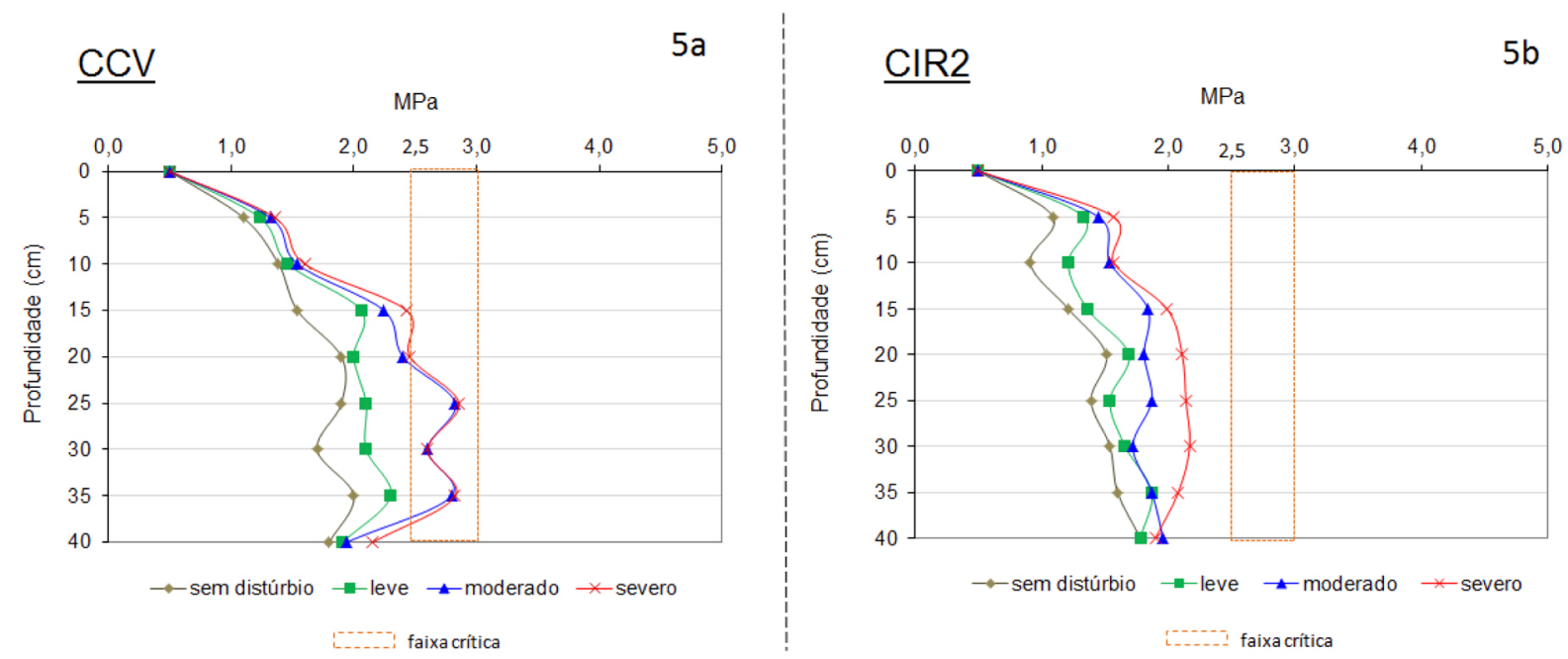

Figura 5. Índice de cone avaliado na Etapa 1 (colheita de lenha) para os tratamentos CCV (5a) e CIR2 (5b).

Figure 5. Cone index evaluated in Step 1 (wood harvesting) for CCV (5a) and CIR2 (5b) treatments.

Conforme apresentado nos gráficos de índice de cone na Figura 6, após a colheita total de Eucalyptus, nota-se que nas áreas com distúrbio superficial moderado e severo dos três tratamentos os valores de resistência do solo à penetração superaram o limite crítico nas camadas mais profundas. Os maiores valores médios de resistência à penetração ocorreram nos dois tratamentos que utilizaram tração mecânica das duas etapas de colheita, sendo de 3,2 MPa no CCV e 3,6 MPa no CIR1. No caso do tratamento de colheita de impacto reduzido CIR1, a compactação foi ainda maior devido à concentração obrigatória do tráfego do trator nos ramais de extração e o consequente aumento do número de passadas pelo mesmo local. Seixas et al. (2003) também constataram maior compactação pela maior intensidade de tráfego numa área com características semelhantes de solo e de topografia.
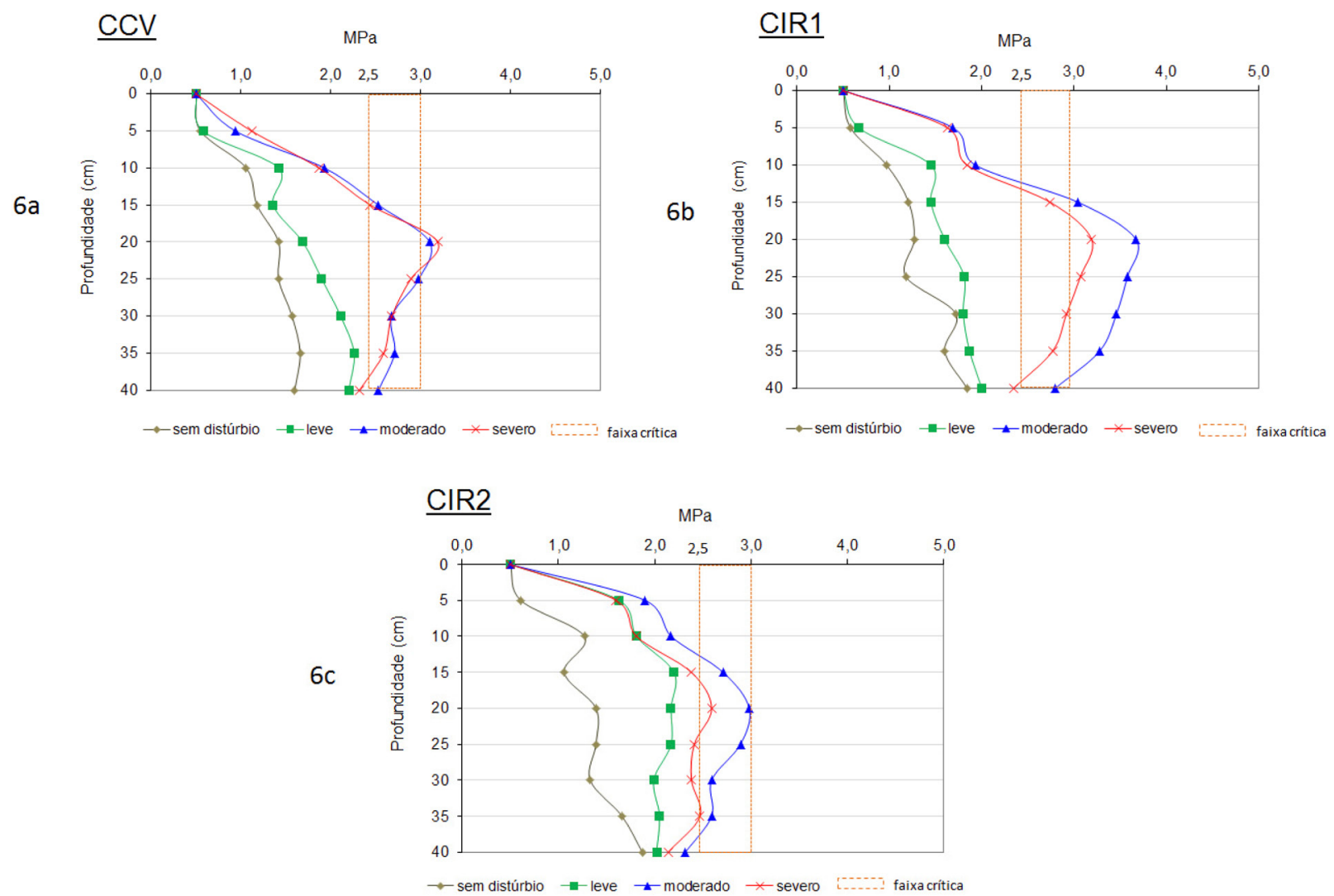

Figura 6. Índice de cone avaliado na Etapa 2 (colheita de tora) para os tratamentos CCV (6a), CIR1 (6b) e CIR2 (6c). Figure 6. Cone index evaluted in Step 2 (log harvesting) to CCV (6a), CIR1 (6b) e CIR2 (6c) treatments. 
Alguns estudos realizados em solos com características semelhantes ao dessa pesquisa inferiram um tempo de resiliência à compactação de 4 a 7 anos (DEDECEK, GAVA, 2002; HAMMEL, 1994 apud SILVA et. al., 2008). Considerando que o poder natural do solo de reverter à compactação depende da associação ao longo do tempo de vários fatores no sítio, como por exemplos, aporte de matéria orgânica e atividade de microrganismos, pode-se inferir que quanto menor a área com distúrbios moderado e severo melhores serão as condições de sítio para reverter à compactação do solo.

Gonçalves (2002) afirmou que a extensão total de área compactada e a profundidade da compactação são fatores que determinarão o grau de compactação prejudicial ao desenvolvimento das plantas. Considerando que o sistema de colheita convencional promoveu maior área por hectare com distúrbios moderado e severo e com níveis de compactação além do limite crítico, é certo afirmar que esse sistema de colheita apresentou um grau de compactação prejudicial ao desenvolvimento da vegetação remanescente no sub-bosque bem maior do que os sistemas de colheita de impacto reduzido.

\section{CONCLUSÕES}

Para a colheita de madeira na forma de lenha, o sistema de impacto reduzido com extração animal (CIR2) mostrou ser alternativa viável porque não promoveu compactação do solo até o nível crítico teórico para o desenvolvimento de plantas.

O sistema de impacto reduzido que utilizou extração mecânica nas duas etapas de colheita (CIR1) manteve uma área significativamente maior nas condições naturais (sem distúrbio) e, por isso, é o mais indicado quando o objetivo for extrair madeira tanto na forma de lenha como de tora.

Os sistemas com colheita de impacto reduzido promoveram significativa redução de área impactada por hectare com distúrbio superficial moderado e severo e, consequentemente, menor área por hectare com níveis de compactação do solo acima do limite crítico teórico para o desenvolvimento de plantas.

Considerando ter sido constatado reduções significativas dos efeitos negativos nos atributos físicos do solo nos tratamentos com colheita de impacto reduzido, concluiu-se que esse sistema é alternativa viável do ponto de vista ambiental para a extração de madeira em talhões abandonados de Eucalyptus e para promover o manejo florestal sustentável em reserva legal.

\section{REFERÊNCIAS BIBLIOGRÁFICAS}

AKAY, A. E.; YILMAZ, M.; TONGYE, F. Impact of mechanized harvesting machines on forest ecosystem: residual stand damage. Journal of Applied Sciences, Dubai, v. 6, n. 11, p. 2414-2419, 2006.

BARROS, A. C.; UHL, C. Logging along the Amazon River and estuary: patterns, problems and potential. Forest Ecology and Management, Amsterdan, v. 77, p. 87-105, 1995.

BRASIL. Lei n. 12.651, de 25 de maio de 2012. Dispõe sobre a proteção da vegetação nativa. Disponível em: < http://www2.camara.gov.br/legin/fed/lei/2012/lei-12651-25-maio-2012-613076-norma-pl.html >. Acesso em: 23 jul. 2012.

CARNEIRO, P. H. M. Caracterização florística, estrutura e da dinâmica de regeneração de espécies nativas em um povoamento comercial de Eucalyptus grandis em Itatinga/SP., 2002. 131 p. Dissertação (Mestrado na área de Ciências Florestais) - Escola Superior de Agricultura "Luiz de Queiroz", Universidade de São Paulo, Piracicaba, 2002.

CLATTERBUCK, W. K. Logging damage to residual trees following comercial harvesting to diferente retention levels in a mature hardwood stan in Tenessee. Asheville, NC: U.S. Department of Agriculture, Forest Service, Southern Research Station, 2006. 594 p. (Gen. Tech. Rep. SRS-92)

DEAL, J. A. Site disturbance and social impacts of animal logging and conventional logging in Appalachia. Blacksburg, 2002. Disponível em: < http://healingharvestflorestfoundation.org/docs/Joshua\%20Deal\%20 Abstract.doc >. Acesso em: 06 jun. 2012.

Sci. For., Piracicaba, v. 46, n. 118, p. 143-155, jun. 2018 DOI: dx.doi.org/10.18671/scifor.v46n118.01 
Mendes et al. - Avaliação dos impactos da colheita florestal nos atributos físicos do solo em uma reserva legal

DEDECEK, R.A.; GAVA, J.L. Influência da compactação do solo na produtividade da rebrota de eucalipto. Revista Árvore, Viçosa, v. 29, n. 3, p. 383-390, 2005.

FAO - FOOD AND AGRICULTURE ORGANIZATION OF THE UNITED NATIONS. Reduced impacto logging in tropical forests: literature synthesis, analysis and prototype statistical framework. Roma: FAO, 2004.8 p. Disponível em: < ftp://ftp.fao.org/docrep/fao/008/j4290e/j4290e00.pdf >. Acesso em: 01 mai. 2011.

FICKLIIN, F. L.; DWYER, J. P.; CUTTER, B. E.; DRAPER, T. Residual tree damage during selection cuts using two skidding systems in the Missouri Ozarks. In: CENTRAL HARDWOOD CONFERENCE,11., 1997, Columbia. Proceedings... Columbia: USDA, 1997. Disponível em: < http://www.nrs.fs.fed.us/pubs/gtr/other/gtr nc188/ CHvolume11page036.pdf >. Acesso em : 6 jun. 2012.

GONÇALVES, J. L. M. Principais solos usados para plantações florestais. In: GONÇALVES, J. L.; STAPE, J. L. (Ed.). Conservação e cultivo de solos para plantações florestais. Piracicaba, SP: IPEF, 2002, cap. 1, p. 1-46.

GONÇALVES, J. L. M.; ALVARES, C. A.; GONÇALVES, T. D.; MOREIRA, R. M.; MENDES, J. C. T.; GAVA, J. L. Mapeamento de solos e da produtividade de plantações de Eucalyptus grandis, com uso de sistema de informação geográfica. Piracicaba, SP. Sciencia Forestalis, Piracicaba, v. 40, n. 94, p. 187-201, 2012.

GONÇALVES, R. M. G.; LUCA, E. F.; ZANCHETTA, D.; FONTES, M. A. L. Fitossociologia do estrato arbóreo e arbustivo em sub-bosque de talhões de Pinus elliottii e Eucalyptus maculata/citriodora na Estação Experimental de Tupi, Piracicaba - SP. Revista Instituto Florestal, São Paulo, v. 22, n. 2, p. 259-277, 2010.

JOHNS, J. S.; BARRETO, P.; UHL, C. Os danos da exploração madeireira com e sem planejamento na Amazônia Oriental. Belém: IMAZON, 1998. 40 p. (Série Amazônia, 16)

HOLMES, T. P.; BLATE, G. M.; ZWEEDE, J. C.; PEREIRA JUNIOR, R.; BARRETO, P.; BOLTZ, F. Custos e benefícios financeiros da exploração florestal de impacto reduzido em comparação com a exploração convencional na Amazônia Oriental. 2. ed. Belém: Fundação Floresta Tropical/Instituto Floresta Tropical (IFT), 2004. 68 p.

LOPES, E. S.; OLIVEIRA, D.; RODRIGUES, C. K.; DRINKO, C. H. Compactação de um Solo Submetido ao Tráfego do Harvester e do Forwarder na Colheita de Madeira. Floresta e Ambiente, Seropédica, v. 22, n. 2, p. $223-230,2015$.

MALINOVSKI, J. R.; CAMARGO, C. M. S.; MALINOVSKI, R. A.; MALINOVSKI, R. A. Sistemas. In: MACHADO, C.C. (Ed.). Colheita Florestal. 2. ed. Viçosa: UFV, 2008. p. 161-184.

MEADOWS, J. S. Logging damage to residual trees following partial cutting in a Green Ash-Sugarberry stand in the Mississipi delta. In: GILLESPIE, A. R.; PARKER, G. R.; POPE, P. E.; RINK, G. (Ed.) In: CENTRAL HARDWOOD FOREST CONFERENCE., 9., 1993. St. Paul. Proceedings...St Paul: USDA, 1993. p. $248-260$.

MINETTI, L. J.; OlIVEIRA FILHO, R. F; PINTO, L. A. A.; SOUZA, A. P.; FIEDLER, N. C. Análise técnica e econômica do corte florestal planejado de floresta tropical úmida de terra-firme na Amazônia Ocidental. Revista Árvore, Viçosa, v. 24, n. 4, p.429-435, 2000.

ONOFRE, F. F. Restauração da Mata Atlântica em antigas unidades de produção florestal com Eucalyptus saligna Smith. no Parque das Neblinas, Bertioga, SP. 2009. 120 p. Dissertação (Mestrado na área de Recursos Florestais) - Escola Superior de Agricultura “Luiz de Queiroz”, Universidade de São Paulo, Piracicaba, 2009.

PIMENTEL-GOMES, F; GARCIA, C.H. Estatística aplicada a experimentos agronômicos e florestais: exposição com exemplos e orientações para uso de aplicativos. Piracicaba: FEALQ, 2002. 309 p. 
PINTO, A. C. M.; COUZA, A. L.; SOUZA, A. P.; MACHADO, C. C.; MINETTE, L. J.; VALE, A. B. Análise de danos de colheita de madeira em floresta tropical úmida sob regime de manejo florestal sustentado na Amazônia Ocidental. Revista Árvore, Viçosa, v. 26, n. 4, p. 459-466, 2002.

RODRIGUES, R. R.; BRANCALION, P. H. S.; ISERNHAGEN, I. (Org.). Pacto pela restauração da mata atlântica: referencial dos conceitos e ações de restauração florestal. São Paulo: Instituto BioAtlântica, 2009. 256 p. Disponível em: < http://www.pactomataatlantica.org.br/pdf/referencial-teorico.pdf >. Acesso em: 20 out. 2011.

SABOGAL, C.; SILVA, J. N. M.; ZWEED, J.; PEREIRA JUNIOR, R.; BARRETO, P.; GUERREIRO, C. A. Exploração de impacto reduzido em operações florestais de terra firme na Amazônia Brasileira. Belém: EMBRAPACIFOR, 2000. 8 p.

SAPORETTI, A. W.; NETO, J. A. A. M.; ALMADO, R. Fitossociologia de sub-bosque de cerrado em talhão de Eucalyptus grandis W. Hill ex Maiden no município de Bom Despacho-MG. Revista Árvore, Viçosa, v. 27, n. 6, p. 905-910, 2003.

SEIXAS, F. Efeitos físicos da colheita mecanizada de madeira sobre o solo. In: GONÇALVES, J. L. M.; STAPE, J. L. (Ed). Conservação e cultivo de solos para plantações florestais. Piracicaba: IPEF, 2002. p. 313-350.

SEIXAS, F;; SOUZA, C. R. Avaliação e efeito da compactação do solo, devido à freqüência de tráfego, na produção de madeira de eucalipto. Revista Árvore, Viçosa, v. 31, n. 6, p. 1047-1052, 2007.

SEIXAS, F; OLIVEIRA JUNIOR, E. D. Compactação do solo devido ao tráfego de máquinas de colheita de madeira. Scientia Forestalis, Piracicaba, n. 60, p. 73-87, 2001.

SEIXAS, F.; KOURY, C. G. G.; RODRIGUES, F. A. Determinação da área impactada pelo tráfego de Forwarder com uso de GPS. Scientia Forestalis, Piracicaba, n.63, p.178-187, 2003.

SEIXAS, F; OLIVEIRA JUNIOR, E.D.; SOUZA, C.R. Efeito da camada de resíduos florestais na compactação do solo causada pelo transporte primário de madeira. Scientia Forestalis, Piracicaba, n. 54, p. 9-16, 1998.

SILVA, S. R.; BARROS, N. F.; COSTA, L. M.; LEITE, F. P. Soil compaction and eucalyptus growth in response to forwarder traffic intensity and load. Revista Brasileira de Ciência do Solo, Viçosa, v. 32, p. 921-932, 2008.

STOLF, R. Teoria e teste experimental de formulas de transformação dos dados de penetrômetro de impacto em resistência do solo. Revista Brasileira de Ciência do Solo, Campinas, v. 15, p. 229-235, 1991.

SZYMCZAK, D. A.; BRUN, E. J.; REINERT, D. J.; FRIGOTTO, T.; MAZZALIRA, C. C.; LÚCIO, A. D. C.; MARAFIGA, J. Compactação do solo causada por tratores florestais na colheita de Pinus taeda L. na região sudoeste do Paraná. Revista Árvore, Viçosa, v.38, n.4, p.641-648, 2014.

VALLE, D.; PHILLIPS, P.; VIDA, E.; SHULZE, M.; GROGAN, J.; SALES, M.; GARDINGEN, P. Adaptation of a spatially explicit individual tree-based growth and yield model and lont-term comparison between reducedimpact and conventional logging in eastern Amazônia, Brazil. Forest Ecology and Management, Amsterdan, v. 243, n. 2-3, p. 187-198, 2007.

Recebido em 08/07/2016

Aceito em 18/10/2017 
